mongrengrenge, et par elle, les anodes sont continuellement en roúsence de surfaces fraiches de mercure.

La solution de sel marin reçoit aussi un mouvement de rotaLa solui se communique ultérieurement au mercure, ef ce delnier, en rajson des rainures radiales creusécs dans le fond de la nier, en rasson dest contmuellement dirigé contre les paros de cette cure

cuve. liextrieur de la cloche se trouve une conche d'eau sur to mercure, qui agit sur l'amalgame et produl la soude caustique, mee dégagement dhydrogène.

clte solution esl maintenue jusqu'a ce qu'elle soit suffisamment concentrée; elle est alors ratirée par un siphon et remplacée par de leau fratche.

les inventeurs ont prévu cque la cuve serant chaulfée jusqu'a une lempérature voisine du point d'évaporation des solutions, cela friml avorr une solution de sel marin plus concentrée et aussi pour audmenter l'attaque de l'amalgame et diminuer la résistance de lappareil.

Cefte prévision élait cependant une erreur, car au-dessus de Ti derrés 1 l se forme du chlorate en grande quantité, les anodes soit détruites et il y a une grande chance d'explosion à causc du mólango de chlore et doxygène qui se forme.

Le fait récl est que le passage de 900 ampires, par exemple, dans un tel appareil de 3 pieds de diamètrc, chauffe le liquide en mison de sa propre résistance, plus qu'il ne convient, et que le probleme serait plutot de refroidir l'appareil que de lo chauffer.

La différence de potentiel théorique nécessaire à la décomposilion d'une solution saturée de sel marin, pour former du sodium et dégager du chlore est $\frac{97200}{23040}=4,22$ volts (97200 étant la chaleur de formation du chlorure de sodium en solution concentrée af 23040 la quantité de chaleur correspondant à 1 volt). Toutefis, l'absorption du sodium par le mercure et la formation d'amalginic dégaçe une quantité considérable d'énergie qui diminue le voltage théorique de $\frac{21600}{23040}=0,89$ volts (21 600 étant la chaleur de formation de l'amalgame). Le voltage nécessaire pour la dé composition du sel en solution concentrée est finalement de 3,33 volts. Le voltage pratique actuellement établi pour forcer un couranl de 000 ampères à traverser l'appareil est de 5 volts, dont 1.67 est nécessaire pour vaincre la résistance ohmique de l'appareil el représente l'énerqie électrique convertic en chaleur.

C'est l'équivalent de $\$ 5$ calories-livres par minute dans l'appareil. et c'est suffisant pour chauffer son contenu d'environ $20^{\circ} \mathrm{F}$. en une heure, en ne supposant aucune perte par radiation. Nous vovons, par conséquent, qu'il est tout à fait probable que l'appareil atteindra $20^{\circ} \mathrm{F}$. au-dessus de la température extérieure simplement du fait de la chaleur dégagée par le passage du courant. Ilans un tel appareil il est probable qu'il y aura quelque perte du lait de la recombinaison du sodium dans le compartiment anodique.

On n'a prévu aucun dispositif spécial pour le renouvellement de l'amalgame ni aucune methode pour le déchar'ger au dehors.

Ces deux opérations se produisent par elles-memes, pour ainsi dire, a la suite de l'action de charge.

M. Rhodin a établi que la perte résultant de la redissolution au contact du sel étail de $3 \%$

On pouvait s'atlendre à ce qu'elle fủt plus grande. La déchargo est voltaique de sa nature; car la cuve en fer et l'amalgame forment ur couple voltalque fermé en court-circuit, qui, au conlact de l'eau, entrâne la décharge du sodium et le dégagement de liydrogène au contact du fer.

Cette action diffère de la méthode de décharge de lamalgame de Reller, en ce que cette dernierc utilise une plaque cathodique disincte dans la solution caustique, séparée de l'amalgame et non en contact physique, cel ensemble constituant une pile galvanique dont l'amalgame est un pole, et la cathode l'autre pole, et de laquelle un courant galvanique peut être produit et utilisé, ainsi que le fait Keller d'après son brevet allemand, soit pour renforcer le eourant principal de aécomposition, soil pour toute autre action indépendante.

Avec l'action du couple voltaique de Rhodin, la force électromotrice engendrée n'est pas utilement applicable; l'énergie tout enthre de la décomposition de l'amalgame, représentant le cinparme de l'énergie électrique absorbée par l'appareil, surchauffe

a soude caustique et augmente ainsi la tomperature de l'appareil.

L'arrangement adopté par Kellner et Castner, est tel que la

ferce électromotrice de decharge est rendue au circut. Mais une

dans de l'énergie 'récuperée par ce fait est convertie en chaleur

dans la soude caustique pour vaincre la résistance ohmique entre

ande ct la cathode correspondant a cette solution; ajoutons

qu'avec une bonne disposition de cathode, une partie de la force

Linstalrice de décharge reste encore disponible.

ment solation complète comprend 120 appareils, mais 80 seule-

liusine mis en action a la fois.

Lusine de la poudre à blanchir comprend 10 chambres de
55 pieds 6 pouces de long, 17 pieds de large et 6 pieds 6 pouces de haut, constutuées de feuilles de plomb $\mathrm{n}^{\circ} 6$, avec un plancher un luile d'un demi-pouce. L'usme à chaux peut fournir lí tonnes de chaux éteinte par jour.

$\Lambda$ vec 80 apparels en achon, la production est de 1,4 tonnes de poudre $i$ blanchir et 2 tomnes de' soude caushque par jour.

Cecl représente onviron $80 \%$ de la production theorique rap. portée au courant dépensé.

Le sel employé contient $99,5 \%$ de chlorure de surlium, le reste ctant en grande partie consliluee par des sulfates de calemum et de sodium.

Ces sels, en raison de leur décomposition ef le chlore libre entrainent la dissolution de 1 à 1,20 pour cent de mercure dans la solution de sel marm, mais comme la solution cet sans cesse on travail, sa concentration étant mainlenue constante par l'nlimcttation de sel nowvau, la perte résultant de ce fait rat faible, satuf Iorsque la solution est changé, lorsqu'elle devient trop impure, et alors, chaque tros ou quatre huilaines environ, 20 livros de mercure (70 francs) sont perdus. Dans la solution alcaline une livre de morcure (valeur 3 fr. 50 ) esl dissoule nour 27500 livres de produit causlique. Cette perte est donc négligeable au point do vue commercial.

Avec les 120 appareds on action, on arrvera à produire 4 lomnes de soude caustique et 9 tonnes de poudre a blanehir par jour; celie derniere quantité sufira ì la consommation du Canada: li premiere a une grande parlic de cotte meme consommation, mats élant domé le pix de ces moduls sur lo marché au Cimada, l'usme ne saurat se maintenr avec profit, sans un larif de protection.

Nous apprenons, et nous sommes heureux d'en informer nos lecteurs que l'exploitation des compteurs electriques stanicy "pour courants alternatifs, dont la description detallee a été donnéc dans le numbo io décembre 1903 de la Revue, vient d'utre confié pourla krance à la liompagne Westinghouse. C'est un nouveau gage de succes pour ces appareils déjà très répandus à l'ćtranger.

\section{La Houille blanche en Italie}

Dans le numéro de février 1904 , La Houille Blanche a donné une «Statistique des concessions d'eau en Italie » qui se rapportait à l'ensemble des concessions accordées par l'Etat dans tout le pays. Nous nous proposons aujourd'hui de donner ici le résumé d'un rapport que le Ministère de l'Agriculture, de l'Industrie et du Commerce de Rome vient de faire paraître sur les richesses en énergie hydraulique de l'Italie centrale. On pourra ainsi apprécier l'importance des disponibilités de houille blanche dont on pourrait tirer parti dans cette région.

Ce rapport évalue à $300000 \mathrm{HP}$ la puissance utilisable du Tibre; sur ce chiffre, on ne prévoit encore l'emploi que de 100 ooo HP. D'autre part, le Garigliano, le Volturno, le Sarno, le Tosciano, le Scle seraient capables de fournir 180 ooo HP sur lesquels on n'utilise actuellement que 35000 HP. De plus, on pourrait tirer des rivières Marna, Flora, Lombrone et Tronto $2 \mathrm{I} 2$ ooo HP, alors que l'on met à profit, actuellement, à peine la dixième partie de cette dernière puissance.

Le même rapport évalue à 767000 HP au total la puissance que l'on peut emprunter aux cours d'eau de l'Italie centrale. Comme il reste encore une disponibilité de 592000 $\mathrm{HP}$, on se rend facilement compte dés conséquences économiques que peut avoir, pour l'Italie, dont le sous-sol est très pauvre en houille noire, la mise en valeur de forces naturelles aussi abondantes.

Nous donnerons sous peu le résumé d'une conférence faite à la section milanaise de I'Association Electrotechnique Italienne sur des phénomènes d'instabilité observés dans des couplages d'alternateurs et de moteurs synchrones. 


\section{Indicateur à distance de Niveau d'eau}

\author{
Système Siemens et Halske
}

Il est souvent aussi commode qu'utile de pouvoir connaître dans un endroit déterminé, par excmple dans le bureau d'un ingénicur ou dans une salle de laboratoire ou de machines, quelle est à un instant quelconque la position relative du niveau de l'eau ou de tout autre liquide, dans un réservoir d'alimentation, une chambre de mise en charge, un canal ou une rivière situés à une certaine distance. C'est pourquoi nous nous permettons de présenter aux lecteurs de cette Revue la description de l'indicateur-enregistreur

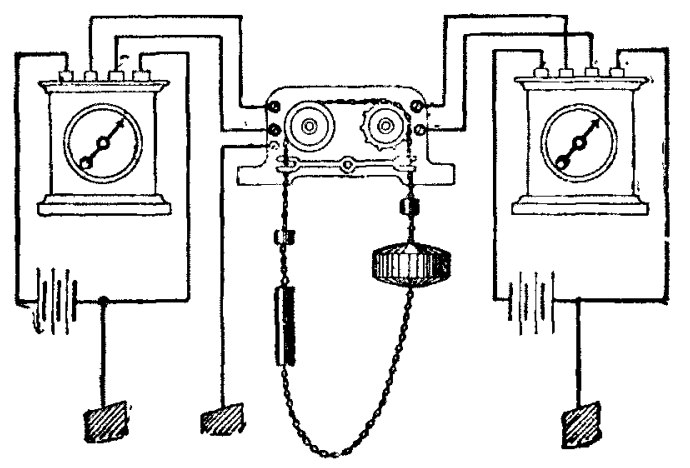

suivant qui a fait ses preuves dans de nombreuses installations et dont MM. Rousselle et Tournaire, de Paris, sont seuls concessionnaires pour la France.

L'ensemble du systeme d'indicateur à distance se compose de trois parties bien distinctes: le transmetteur, la ligne de transmission et enfon le récepteur. Il est bien évi. dent que dès que la distance dépasse quelques métres, une transmission électrique est seule pratique, aussi c'est à elle qu'on a eu recours.

Transmetteur. - Le transmetteur consiste essentiellement en un flotteur qui est supporté par une chaine et en un dispositif de contact qui est enfermé dans une boìte en fonte complètement étanche qui le maintient à l'abri de l'humidité. La chaîne qui est constamment tendue par un contrepoids passe sur une roue dentée qu'elle entraîne dans un sens ou dans l'autre suivant que l'eau monte ou descend. Dans le type courant, l'appareil indique chaque varation de niveau de 5 centimetres.

Pour cela, au fur et à mesure que le niveau descend, le flotteur entraine la chaine et fait tourner la rove dentée ; lorsque le flotteur est descendu de 5 centimètres, la roue dentée a tourné d'un tiers de tour et est venue actionner un butoir qui ferme le circuit d'une batterie de piles dont le courant va actionner les électros du récepteur. Lorsque le courant est lancé, un déclic ramène le butoir à sa position première, tandis que la roue dentée peut continuer son mouvement. Apres une nouvelle descente de 5 centimètres, la roue dentée a encore tourné d'un tiers de tollr et de nouveau actionné le butoir qui lance un second courant de même sens que le premier, et ainsi de suite. Si le niveau monte au contraire, le contrepoids entraîne la chaîne qui, à son tour provoque la rotation en arriere de la roue dentée et, par un dispositif analogue au précédent mais inversement placé, actionne un nouveau butoir qui ferme encore le circuit de la batterie de piles et envoie dans la ligne un courant de sens inverse des précédents. La chaîne est en outre munie de deux poids qui, lorsque les niveaux maximum ou minimum sont atteints, viennent rencontrer une double fourche située aundessous de la roue dentée, ce qui a pour effet de provoquer un signal avertisseur spécial au moyen d'une sonnerie installée dans le poste récepteur.

L'appareil transmetteur se place immédiatement au dessus du réservoir, sur un support adapté aux conditions locales, par exemple sur une planche, une console ou une poutre. Dans tous les cas il doit être monté de telle sorte que le flotteur et son contrepoids plongent librement dans l'eau et à un endroit où ils ne soient soumis à l'action d'aucun remous; s'il n'y avait pas moyen d'éviter ces remous, il faudrait placer le flotteur dans un tube assez large servant d'abri.

Ligne de transmission. - Cette ligne se compose de deux fils d'aller, aériens ou souterrains, et d'un retour parla terre; toutefols il est bon, lorsqu'on se sert de fils souter. rains, d'employer un cable à trois brins isolés dans lequelle troisième brin sert de fil de réserve.

Dans le cas d'une ligne aérienne on utilise avec avantage du fil de fer galvanisé de 3 millimètres de diamètre ou de préférence du bronze siliceux de 2 millimètres. Il est bon de mettre les deux fils l'un au-dessus de l'autre, pour éviter qu'ils ne viennent à se toucher sous l'action du vent. Lorsqu'on se sert de câbles souterrains on les place dans une tranchée de 30 à 40 centimètres de profondeur en avant soin de se tenir à une certaine distance des canalisations vo:

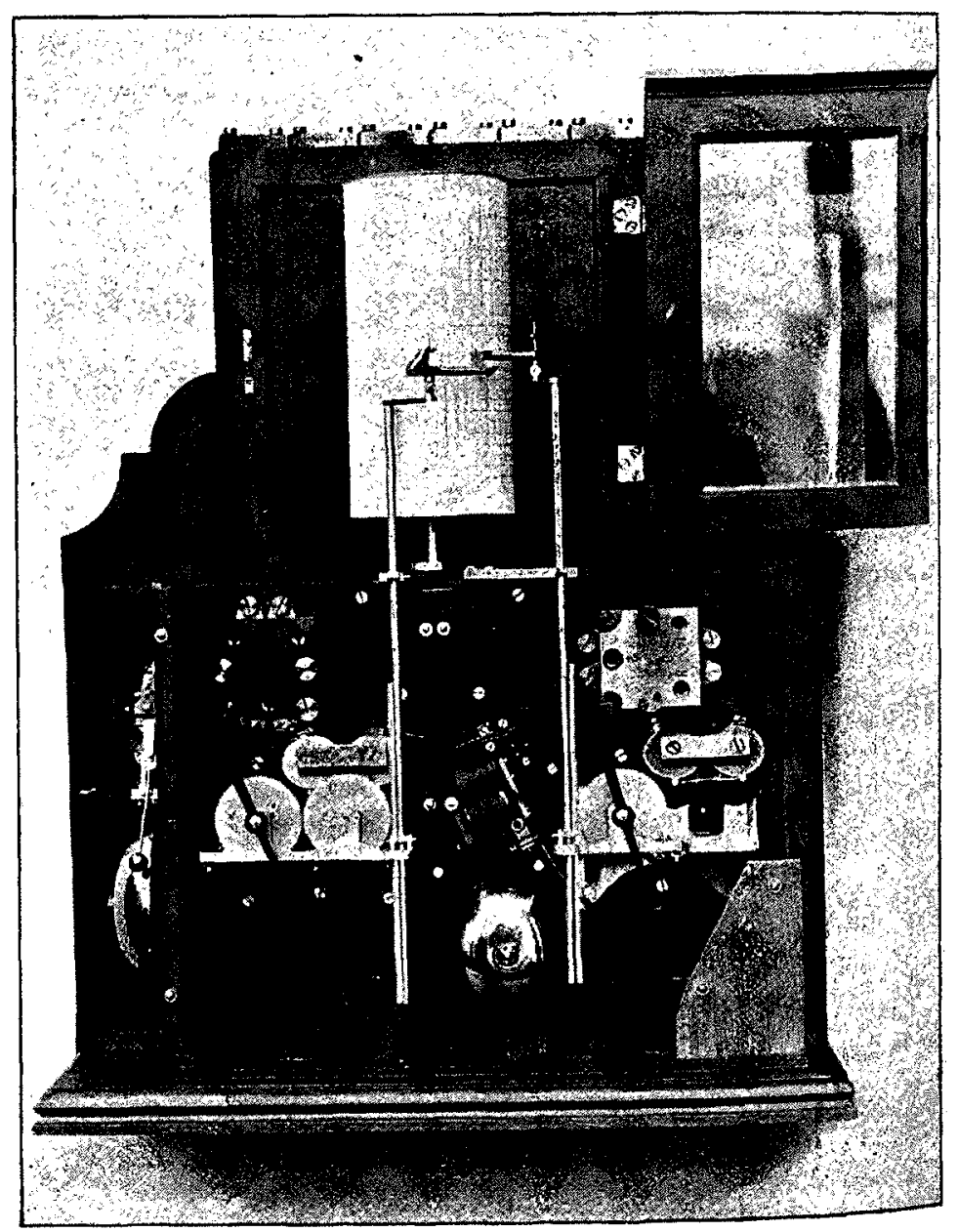

VUE D'UY INDICATEUR-ENREGISTREUR

sines. Enfin, à l'extrémité de la ligne, à l'entrée du posterécepteur, on dispose un parafoudre analogue à ceux qui sont employés dans les services télégraphiques ou téléphoniques et que l'on relie à la terre.

Les conducteurs qui relient les appareils et le parafoudre a la terre doivent avoir une résistance électrique aussi faible 
que possible et être soudés aux résèaux des canalisations d'eau ou de gaz s'il y, en a,' ou à leur défaut à des plaques de fonte enfouies dans un sol humide.

Le courant électrique nécessaire est fourni à la ligne par une batterie de piles : un indicateur avec une ligne double de un kilomètre de longueur nécessite environ dix éléments Leclanché ; le même appareil avec un enregistreur en demande douze. Si la distance est supérieure à un kilomètre, il faut ajouter deux éléments de plus par kilomètre, dans le cas de fils de fer 'galvanisés de 3 millimètres ou seule. ment un élément avec le bronze siliceux de 2 millimètres. chaque variation de niveau de 5 centimetres, mais il est facile, sans rien changer à l'intérieur du transmetteur, de pouvoir enregistrer au besoin des variations beaucoup plus faibles.

Avec un seul poste transmetteur on peut actionner plusieurs indicateurs ou enregistrcurs que l'on disposera, soit en série, soit en dérivation comme le montre le schéma précédent, suivant les besoins du service ou les conditions particulières dans lesquelles on se trouvera.

\section{J. RACINE,}

Licencie ès-Sciences.

\section{Wasserwerk Trachan.}
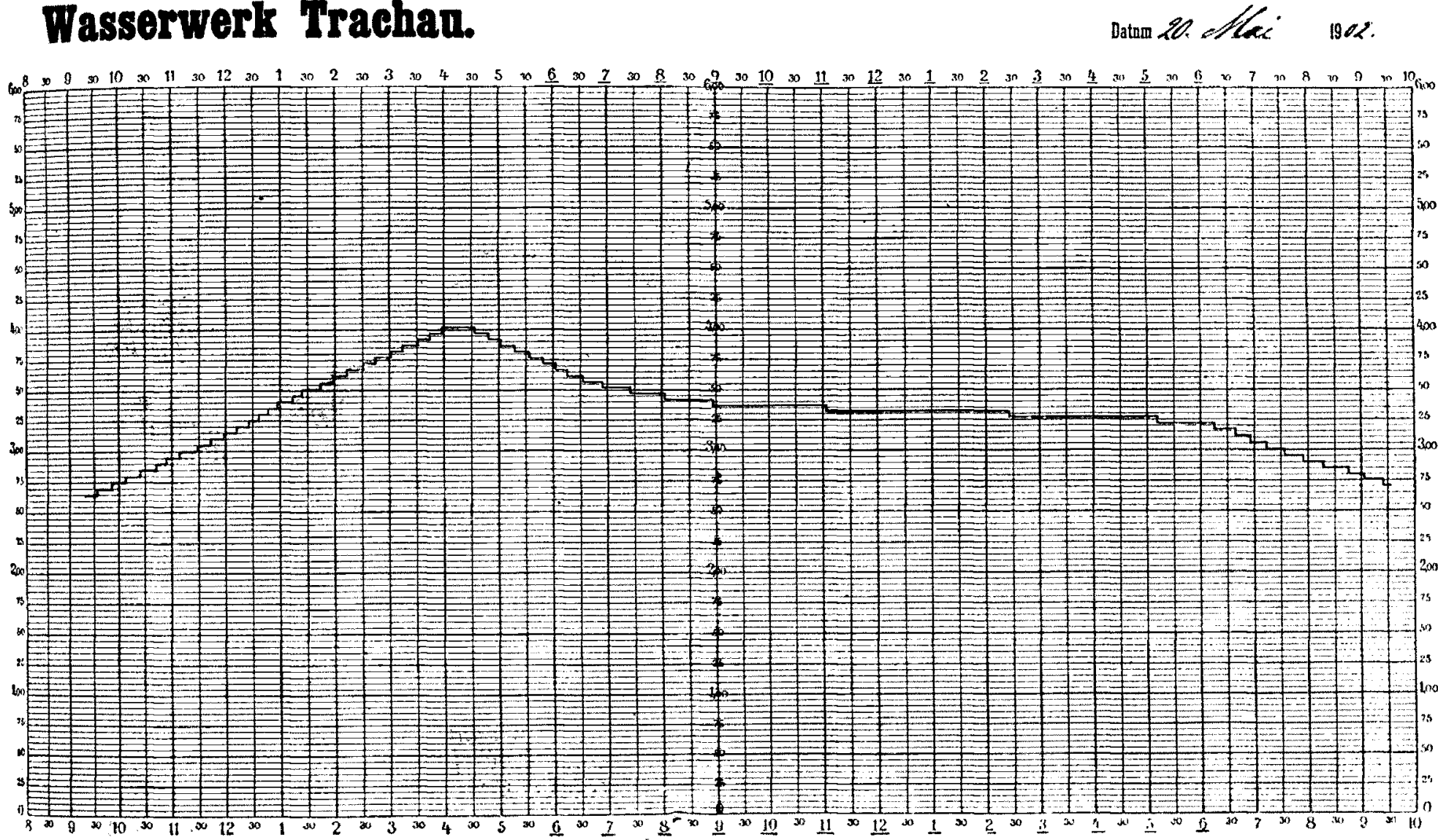

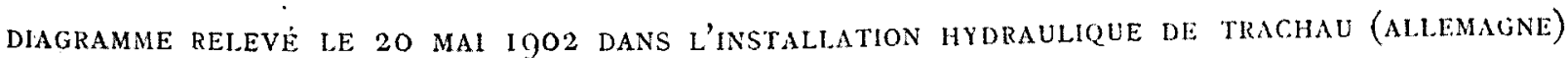

\begin{abstract}
Récepteur. - Le récepteur qui peut être un simple indicateur ou bien un en registreur est actionné par deux paires d'électro-aimants décalées de 120 degrés l'une par rapport à lautre. Ces électros agissent sur une palette munie d'un côté d'un contrepoids et pouvant tourner autour d'un axe horizontal. Cette palette transmet son mouvement au cadran de l'indicateur au moyen d'une vis sans fin et d'un pignon hélicondal. Chacune des deux paires d'électros est reliée à l'un des fils de la ligne de transmission de telle sorte qu'à chaque contact du transmetteur la palette de l'indicateur tourne de 120 degrés d'un côté ou de l'autre, suivant que le niveau monte ou qu'il baisse. Dans le cas d'un récepteur enregistreur la palette transmet son mouvement, par le moyen d'une petite crémaillère, à une plume qui trace la courbe des variations du niveau de l'eau sur un cylindre enregistreur tournant avec une vitesse uniforme au moyen d'un mouvement d'horlogerie.

Nous donnons ici le relevé d'un diagramme journalier inscrit le 20 mai 1902, dans l'installation hydraulique de Trachau (Allemagne). Comme nous l'avons fait remarquer précédemment, l'appareil du type courant enregistre
\end{abstract}

\section{LE MOIS HYDRO-ÉLECTRIQUE}

\section{INFORMATIONS DIVERSES}

\section{Conférence de M. Alliévi sur les coups de bélier dans les conduites fermées.}

Le 8 mai 1804 , a eu lieu dans la salle des conférences de la Chambre de Commerce de Grenoble et sous les auspices de la Société pour le développement de l'Enseignement technique près l'Université de Grenoble, la conférence de M. l'ingénicur AlLIÉvi sur " les coups de bélier dans les conduites fermées 》. A cette conférence se pressaicnt un grand nombre d'ingénieurs et d'industriels des régions lyonnaise, dauphinoise et savoisienne, de professeurs de l'Université de Grenoble et d'éléves ou d'anciens élèves de l'Institut Electrotechnique de cette ville.

M. Altí́vi a développé devant un auditoire très intéresséles éléments de sa théorie mathématique du mouvement varić de l'eau dans les conduites, théorie applicable directement aux coups de bélier et permettant de calculer, avec une aisance que rend surprenante l'habileté d'analyste du conférencier, les caractéristiques d'un de ces phénomènes. La Houille Blanche 